\title{
Kompetenzorientierung in der Finanziellen Grundbildung als Grundlage für die Programmentwicklung
}

\author{
Ewelina Mania
}

Eingegangen: 31. März 2015 / Angenommen: 10. Juli 2015 / Online publiziert: 1. September 2015 (C) Die Autor(en) 2015. Dieser Artikel ist auf Springerlink.com mit Open Access verfügbar.

Zusammenfassung Die Gestaltung von Angeboten stellt die zentrale Aufgabe der Programmplanenden in der Erwachsenenbildung dar. Im Bereich Finanzielle Grundbildung gibt es bisher kaum Angebote und didaktische Grundlagen. Daher wird gefordert, Kompetenzanforderungen für Finanzielle Grundbildung zu ermitteln sowie entsprechende Lernangebote zu entwickeln. Kompetenzmodelle, welche die Handlungsanforderungen in bestimmten Situationen strukturieren, können als Grundlage für die Entwicklung von Programmen und Angeboten in der Erwachsenenbildung begriffen werden. Der Beitrag geht der Frage nach, welche Kompetenzanforderungen sich als Grundlage für die Angebotsentwicklung für die Finanzielle Grundbildung empirisch beschreiben lassen. Es wird eine theoretische und empirische Fundierung des entwickelten Kompetenzmodells Finanzielle Grundbildung vorgestellt. Abschließend werden die Potenziale des Modells für die Praxis der Erwachsenenbildung diskutiert und weitere Forschungsbedarfe aufgezeigt.

Schlüsselwörter Finanzielle Grundbildung · Programmentwicklung · Angebotsentwicklung $\cdot$ Didaktik $\cdot$ Kompetenzmodelle

Dieses Vorhaben wird mit Mitteln des Bundesministeriums für Bildung und Forschung unter dem Förderkennzeichen 01AB12009 gefördert. Die Verantwortung für den Inhalt dieser Veröffentlichung liegt bei der Autorin.

E. Mania $(\bowtie)$

Deutsches Institut für Erwachsenenbildung,

Leibniz-Zentrum für Lebenslanges Lernen (DIE) in Bonn,

Heinemannstr. 12-14, 53175 Bonn, Deutschland

E-Mail: mania@die-bonn.de 


\title{
Competence orientation in financial literacy as a basis for program planning
}

\begin{abstract}
An essential task for administrative staff in adult education is to develop and offer courses. In the field of economic education (basic education), there exist only very few offers and didactic material. Hence, there is a great demand to describe requirements concerning competencies to be taught in this area. Such requirements for competencies may serve as a base for the development of courses in adult education. This article explores the questions on which requirements concerning competencies in basic education can be described empirically. It will provide a theoretical and empirical basis for such a model of competencies. In conclusion, the potentials of such a model to be employed in praxis will be discussed and further research questions will be derived.
\end{abstract}

Keywords financial literacy $\cdot$ program planning $\cdot$ basic skills $\cdot$ didactics competency model

\section{Ausgangslage und Problemaufriss}

In jüngerer Zeit wird eine Beschäftigung mit der Begründung, Bestimmung bzw. Strukturierung von Inhalten als zentrale Aspekte einer Didaktik für Erwachsenenbildung gefordert (vgl. Lehner 2013; Frank 2013). ,Eine Didaktik der Erwachsenenbildung bedarf eines inhaltsorientierten Segments, in dem u. a. Fragen der inhaltlichen Auswahl und Aufbereitung theoretisch und empirisch bearbeitet werden" (Lehner 2013, S. 03-6). Die mangelnde bzw. marginale Beschäftigung mit den Inhalten wird zurückgeführt auf die Diskussion über selbstorganisiertes Lernen und Ermöglichungsdidaktik im Zuge des Aufkommens konstruktivistischer Ansätze (vgl. Frank 2013; Schlutz 2005; Siebert 2005). Bremer und Kleemann-Göhring (2011, S. 17 f.) betonen mit Blick auf sogenannte bildungsferne Teilnehmendengruppen die Notwendigkeit, ,sich in der pädagogischen Praxis nicht an (idealisierten) Formen von selbstgesteuertem Lernen zu orientieren“, die für Lernende eine Überforderung darstellen können. Institutionelle Weiterbildung ermöglicht diesen Gruppen systematisches Lernen, das Kompetenzen für die gesellschaftliche Teilhabe vermittelt. Die Angebote müssen so konzipiert sein, dass sie den Nutzenvorstellungen der Adressatinnen und Adressaten entsprechen, um als sinnvoll empfunden zu werden (vgl. Bremer und Kleemann-Göhring 2011, S. 13 f.).

Die Gestaltung von Programmen und Angeboten wird als Bestandteil didaktischen Handelns von professionell Planenden in der Erwachsenenbildung gesehen und untersucht (vgl. Reich-Claassen und von Hippel 2011; Höffer-Mehlmer 2011; Gieseke 2008). Dabei wird das Programmplanungshandeln in der Erwachsenenbildung als „Angleichungshandeln“ (Gieseke 2008, S. 48) und als Vermittlung zwischen den drei Instanzen gesellschaftlicher Bedarf, Adressatinnen und Adressaten sowie pädagogischer Auftrag gesehen (vgl. von Hippel et al. 2008, S. 666). Auch wenn der Erwachsenenbildungsmarkt nachfrageorientiert ist, so hat die Erwachsenenbildung gleichzeitig die Aufgabe, ,auf gesellschaftlich relevante Entwicklungen 
und Zukunftsprobleme frühzeitig aufmerksam zu machen und neue Bildungsbedürfnisse zu wecken“" (Siebert 2006, S. 70).

Der Bedarf an Grundbildungskompetenzen wird derzeit durch die Ergebnisse der aktuellen PIAAC-Studie (vgl. Rammstedt 2013) und der leo. - Level-One Studie (vgl. Grotlüschen und Riekmann 2012) belegt. Die internationale bildungspolitische Bedeutung des Themas Grundbildung bzw. Literacy lässt sich an verschiedenen Initiativen und Förderprogrammen wie der „Nationalen Strategie für Alphabetisierung und Grundbildung“ (BMBF 2012) oder dem „European Literacy Policy Network ELINET“ ablesen. Während frühere Grundbildungskonzepte auf Lesen und Schreiben als Kulturtechnik fokussierten, werden nun verschiedene Kompetenzbereiche unterschieden, wie Gesundheit/Health Literacy, Ernährung/Food Literacy, Politische Grundbildung oder Finanzielle Grundbildung/Financial Literacy (vgl. Egloff 2014, S. 104; BMBF 2012, S. 1). Im Folgenden wird Finanzielle Grundbildung als Teil des Programmbereichs Alphabetisierung und Grundbildung thematisiert.

Zur Legitimation der Notwendigkeit Finanzieller (Grund-)Bildung werden die weltweite Wirtschafts- und Finanzkrise sowie eine „Reihe von sozialen, politischen und ökonomischen Entwicklungstendenzen“ (Aprea 2012, S. 1) angeführt. Beispielsweise wird auf den hohen Verschuldungsgrad von Privathaushalten, die Prekarisierung von Arbeitsverhältnissen, die gestiegene Komplexität von Finanzdienstleistungen und die zunehmende Erfordernis privater Vorsorge verwiesen (vgl. Weber et al. 2013; Remmele et al. 2013; Aprea 2012; Hummelsheim 2010; Reifner 2006; Leinert 2004). Die aktuelle Brisanz des Themas zeigt auch die Aufnahme von Financial Literacy als basale Leistungsdisposition und „essential life skill“ in das PISA-Programm der OECD (2014, S. 118). Hummelsheim (2010, S. 7 f.) formuliert für die Erwachsenenbildung die Aufgabe, grundlegende Kompetenzanforderungen im Bereich Finanzielle Grundbildung zu ermitteln sowie entsprechende niedrigschwellige Lernangebote zu entwickeln.

Hier schließt der folgende Beitrag mit nachstehender Forschungsfrage an: Welche Kompetenzanforderungen lassen sich als Grundlage für die Programm- und Angebotsentwicklung im Bereich Finanzielle Grundbildung empirisch beschreiben? Nach der Vorstellung des Diskussions- und Forschungsstands zu bisherigen Kompetenzbeschreibungen und Angeboten im Bereich Finanzielle Grundbildung wird die theoretische und empirische Fundierung des im Projekt ,Schuldnerberatung als Ausgangspunkt für Grundbildung - Curriculare Vernetzung und Übergänge“" (CurVe) ${ }^{1}$ entwickelten Kompetenzmodells Finanzielle Grundbildung dargelegt. Abschließend werden Potenziale des Modells für die Praxis der Erwachsenenbildung diskutiert und weitere Forschungsbedarfe aufgezeigt.

\section{Diskussions- und Forschungstand}

Im internationalen Diskurs wird seit einigen Jahren zunehmend von „Financial Literacy“, d. h. dem ,adäquaten Umgang mit Geld und Finanzthemen“ (Aprea 2012,

\footnotetext{
${ }^{1}$ Das Projekt ist dem BMBF-Förderschwerpunkt „Arbeitsplatzorientierte Alphabetisierung und Grundbildung Erwachsener" zugeordnet.
} 
S. 1) oder „Financial Capability“ (vgl. u. a. Elliott et al. 2010) gesprochen. In Deutschland reicht das Spektrum der Begriffe von „Finanzieller (Grund-)Bildung“ über „Finanzkompetenz“ bis hin zu „Finanzieller Alphabetisierung“ bzw. ,Finanziellem Analphabetismus“ (vgl. Aprea 2012; Remmele et al. 2013; Leinert 2004).

Kompetenzmodellierungen von Financial Literacy werden bisher vor allem in der Berufs- und Wirtschaftspädagogik, den Wirtschaftswissenschaften und der ökonomischen Bildung/Ökonomie vorgenommen (vgl. u. a. Schürkmann und Schuhen 2013; Seeber et al. 2012). Diese Arbeiten sind jedoch nicht auf dem Grundbildungsniveau angesiedelt, sondern in der Regel auf die Kompetenzentwicklung und -messung von Schülerinnen und Schülern oder jungen Erwachsenen gerichtet. In dem Kompetenzmodell der ökonomischen Grundbildung von Remmele et al. (2013, S. 117 ff.), das Finanzielle Grundbildung als einen Teilbereich vorsieht, werden Grundbildungskompetenzen wie Lesen, Schreiben und Rechnen vorausgesetzt. Ein grundsätzlicher Bezug zu basalen schriftsprachlichen und mathematischen Kompetenzen wird in dem „Financial Capability Framework“ von The Basic Skills Agency and Financial Services Authority (2006) hergestellt, ohne jedoch die konkreten Kompetenzanforderungen zu benennen und empirisch zu begründen. Unter „Finanzieller Grundbildung“ werden im Folgenden die ,existenziell basalen und unmittelbar lebenspraktischen Anforderungen alltäglichen Handelns und der Lebensführung in geldlichen Angelegenheiten“ verstanden (Mania und Tröster 2014, S. 140).

Während unter anderem von Banken und Sparkassen, Schuldnerberatungsstellen und Verbraucherzentralen bereits seit längerer Zeit Angebote zur Finanziellen Bildung, ,in Form von Programmen, Projekten und Materialien mit Lehr-Lernmodulen an Schulen und anderen Bildungseinrichtungen" für Jugendliche und junge Erwachsene existieren (Piorkowsky 2009, S. 43), gibt es in der Erwachsenenbildung kaum Angebote zum Umgang mit Geld explizit auf Grundbildungsniveau (vgl. Ambos und Greubel 2012).

Resümierend lässt sich daher konstatieren, dass es bisher an Kompetenzmodellierungen auf Grundbildungsebene und Lernkonzepten für Adressatinnen und Adressaten der Grundbildung mangelt.

\section{Der theoretische Rahmen}

Legt man ein umfassendes Verständnis von Didaktik zugrunde, das über die konkrete Gestaltung von Lehr-Lernsituationen, also die Mikrodidaktik hinausgeht, geht es unter dem Begriff „Makrodidaktik“ um die Programmplanung und Angebotsentwicklung in der Erwachsenenbildung (vgl. Arnold 2010). Neben der Teilnehmerwerbung, der Zuordnung, Differenzierung und Stufung von Veranstaltungsformaten werden dabei vor allem Entscheidungen über die Zielsetzungen und Themenschwerpunkte von Lernangeboten getroffen (vgl. Siebert 1982). Während die konkreten mikrodidaktischen Entscheidungen meist den Lehrenden überlassen werden, gehören makrodidaktische Entscheidungen zum Aufgabenspektrum der hauptberuflichen pädagogischen Mitarbeitenden sowie Referentinnen und Referenten (vgl. Arnold 2010).

Im Hinblick auf die Spezifika und (Handlungs-)Abläufe der Programmplanung und Angebotsentwicklung in der Erwachsenenbildung gibt es vielfältige Erklärungs- 
modelle, die jeweils unterschiedliche Aspekte und Akteure fokussieren (vgl. Schemmann und Seitter 2015, S. 156 ff.). Unter Rückgriff auf das Planungsmodell für die Angebotsentwicklung von Erhard Schlutz (2006, S. 78 ff.), der wiederum auf die Curriculumtheorie von Saul Robinsohn rekurriert und sie für die Erwachsenenbildung anschlussfähig macht, können die Kompetenzanforderungen in bestimmten Situationen und Lebenslagen zum Ausgangspunkt für die Konzeption von Programmen und Angeboten in der Erwachsenenbildung genommen werden. Dabei geht es darum, Lernziele und -inhalte nicht aus einer abstrakten Bildungsidee abzuleiten, sondern anhand der Analyse von (Handlungs-)Anforderungen in „Lebens- und Verwendungssituationen“ (Schlutz 2006, S. 86) zu ermitteln. Je konkreter sich diese umreißen lassen, desto stärker bestimmt dieser Aspekt die Angebotsgestaltung (vgl. ebd.). Entsprechend der Besonderheit der Erwachsenenbildung gegenüber Schule im Hinblick auf die Freiwilligkeit, die mit der notwendigen Orientierung an den Lebenswirklichkeiten und Nutzenvorstellungen der Adressatinnen und Adressaten von Lernangeboten einhergeht, spielt die Perspektive der (potenziellen) Lernenden „im Planungsmodell eine besondere Rolle“ (vgl. ebd., S. 85). Die Potenziale curricularer Lebensweltanalysen im Bereich der Alphabetisierung und Grundbildung verdeutlichte bereits Paolo Freire (1974), der generative Themen, also typische existenzielle Situationen zum Ausgangspunkt für die Auswahl und Bestimmung von Lerninhalten in seinem einflussreichen Alphabetisierungsprogramm genommen hat (vgl. Siebert 2010; Gomez Tutor 2010).

Das Modell der Angebotsentwicklung von Schlutz (2006) ist durch seine Orientierung an den gewünschten Lernergebnissen sowie den Situations- und Verwendungsbezug an den Kompetenzdiskurs in den Bildungswissenschaften anschlussfähig. Auf der Grundlage der einschlägigen Kompetenzdefinition von Weinert (2001, S. 27 f.) werden die kognitiven (Wissen und Können) und nicht-kognitiven Aspekte wie Überzeugungen und Wertehaltungen sowie motivationale Orientierungen und selbstregulative Fähigkeiten differenziert und analytisch getrennt untersucht (vgl. Baumert und Kunter 2006; Schrader 2010). Kompetenzmodelle bilden das „Bindeglied zwischen dem Kompetenzkonstrukt und den realen Aufgaben“ (Schürkmann und Schuhen 2013, S. 76) bzw. den Kern des „Wissens und Könnens“ (Baumert und Kunter 2006, S. 481) in einem bestimmten inhaltlichen Bereich ab. Im Anschluss an das Kompetenzmodell der ökonomischen Grundbildung von Remmele et al. (2013, S. 115) lässt sich dabei zwischen deklarativem Wissen, das Kenntnisse über Fakten, Sachverhalte und Zusammenhänge umfasst, und dem prozeduralem Wissen, also dem Anwendungs- und Verfahrenswissen, unterscheiden. Die jeweiligen Lernfelder, fachbezogene Kompetenzbereiche oder Lern- und Handlungsbereiche werden als Kompetenzdomänen bezeichnet (vgl. Klieme und Leutner 2006). Dies entspricht dem Konzept der situated literacies von Barton und Hamilton (2003), die von unterschiedlichen Literalitäten in unterschiedlichen Domänen, d. h. sozial strukturierten Kontexten oder Lebensbereichen, ausgehen. Literalität wird dabei als soziale Praxis verstanden, so dass die Bedeutungshorizonte, Anwendungskontexte und Situationen, in denen Schriftsprache gebraucht wird, betrachtet werden (vgl. u. a. Zeuner und Papst 2011; Street 2003; Barton und Hamilton 2003; The New London Group 1996).

Ausgehend von den skizzierten Überlegungen werden Kompetenzmodelle, welche die Handlungsanforderungen in bestimmten Verwendungssituationen strukturie- 
ren, als Grundlage für makrodidaktische Entscheidungen, d. h. für die Konzeption von Programmen und Angeboten in der Erwachsenenbildung, begriffen.

\section{Methodisches Vorgehen}

Im Rahmen des Projekts CurVe wurde ein perspektivverschränkender Ansatz gewählt, um ein möglichst realitätsnahes und umfassendes Bild der alltäglichen Kompetenzanforderungen im Bereich Finanzielle Grundbildung zu erhalten. Vor dem Hintergrund der Forschungsfrage wurden drei Expertengruppen festgelegt, die aufgrund ihrer beruflichen Rolle und persönlichen Erfahrungen jeweils eine besondere Perspektive auf den Gegenstand einnehmen: Adressatinnen und Adressaten von Grundbildungsangeboten, Schuldnerberaterinnen und -berater sowie Weiterbildungspersonal. Unter Experten können mit Gläser und Laudel (2004, S. 9) Personen verstanden werden, die ein spezifisches Wissen über soziale Sachverhalte besitzen, in denen sie agieren.

Um die Perspektive potenzieller Lernenden einzubeziehen, wurden Forschende Lernwerkstätten (vgl. Grell 2006) mit insgesamt 18 Ratsuchenden aus der Schuldnerberatung durchgeführt, wobei die Analyse der Handlungsanforderungen im Bereich Umgang mit Geld in Form einer Gruppendiskussion erfolgte. Durch den Zugang über Schuldnerberatungsstellen ergab sich eine spezifische sozio-demografische Zusammensetzung des Samples: die Mehrzahl der ca. zur Hälfte männlichen bzw. weiblichen Teilnehmenden hatte keinen oder einen niedrigen schulischen bzw. beruflichen Abschluss und war zum Zeitpunkt der Veranstaltung arbeitssuchend oder nicht erwerbstätig; das Alter lag zwischen 26 und 65 Jahren.

Die Perspektive von Schuldnerberaterinnen und -beratern sowie des Weiterbildungspersonals aus dem Bereich Alphabetisierung und Grundbildung (planendes und lehrendes Personal), die durch ihre berufliche Funktion mit alltäglichen Anforderungen im Bereich Finanzielle Grundbildung konfrontiert sind, wurde im Rahmen von insgesamt 24 Experteninterviews berücksichtigt (vgl. Gläser und Laudel 2004).

Sowohl die Experteninterviews als auch die Gruppendiskussionen der Forschenden Lernwerkstätten wurden in Anlehnung an die qualitative Inhaltsanalyse ausgewertet (vgl. Gläser und Laudel 2004). Entsprechend den Vorschlägen von Gläser und Laudel (2004, S. 195) baute das Kategoriensystem auf den theoretischen Vorüberlegungen auf und war zugleich offen, indem das „Kategoriensystem im gesamten Verlauf der Auswertung an die Besonderheiten des Materials angepasst" wurde. Die Auswertung aller Daten, die computergestützt mithilfe der Software MAXQDA erfolgte, zielte nicht auf die Herausarbeitung der Besonderheiten der jeweiligen Perspektive, sondern auf die Rekonstruktion der Komplexität von Kompetenzanforderungen im Bereich Finanzielle Grundbildung.

\section{Empirische Ergebnisse}

Bei der Beschreibung von Kompetenzanforderungen im Bereich Finanzielle Grundbildung lässt sich analytisch zwischen kognitiven und nicht-kognitiven Kompetenzbestandteilen trennen. Die Aussagen in den drei Datensätzen im Hinblick auf die 
Beschreibung der nicht-kognitiven Kompetenzbestandteile im Bereich Umgang mit Geld lassen sich im Anschluss an Baumert und Kunter (2006) den Kategorien Überzeugungen und Wertehaltungen sowie motivationale Orientierungen und Selbstregulation zuweisen. So beeinflussen beispielsweise die Lebensstilvorstellungen die Prioritätensetzung bei Ausgaben:

Also das überhaupt einmal wahrzunehmen, dass materielle Dinge nicht unbedingt alles sind im Leben. Also dass auch so dieses, diese Erwartung, dass man sich alles Mögliche leisten kann, weil es irgendwie oder weil es der Nachbar hat oder sonst wie, nicht? Und dass das erst glücklich macht. Dass das nicht der Maßstab sein kann (I 14, A. 36) ${ }^{2}$.

Im Folgenden werden die kognitiven Kompetenzbestandteile fokussiert, die in einem Kompetenzmodell Finanzielle Grundbildung münden.

Als Ergebnis der Interpretation des Datenmaterials vor dem gewählten theoretischen Hintergrund ist das Kompetenzmodell Finanzielle Grundbildung entstanden, das zum einen sechs Domänen, inklusive 23 Subdomänen, und zum anderen die Dimensionen Wissen, Lesen, Schreiben und Rechnen vorsieht. Im Sinne einer Kreuztabelle bilden diese die Heuristik für die Bestimmung und Verortung basaler (Handlungs-)Anforderungen beim Umgang mit Geld. Um das Zustandekommen der Kategorien zu begründen, wird bei der Darstellung der kognitiven Bestandteile des Modells im Sinne einer ,selektiven Plausibilisierung“ (Flick 1995, S. 169) auf exemplarische Originaltranskripte und -zitate aus dem Datenmaterial zurückgegriffen.

\subsection{Domänen}

Das Kompetenzmodell Finanzielle Grundbildung beinhaltet folgende sechs Domänen Finanzieller Grundbildung:

1. Einnahmen,

2. Geld und Zahlungsverkehr,

3. Ausgaben und Kaufen,

4. Haushalten,

5. Geld leihen und Schulden,

6. Vorsorge und Versicherungen.

Am Beispiel der Domäne Haushalten wird nun anhand der empirischen Daten der Zuschnitt und Inhalt der Domänen dargestellt.

\subsubsection{Haushalten}

Die Kategorie Haushalten bezieht sich grundsätzlich auf die Relation von Einnahmen und Ausgaben. Es geht darum, mit dem vorhandenen Geld ,auszukommen“ (I 3,

${ }^{2}$ Die Transkripte wurden folgendermaßen anonymisiert: I=Interview, Nr. 1-14 im Bereich Schuldnerberatung, Nr. 15-24 im Bereich Weiterbildung; GD=Gruppendiskussion mit Ratsuchenden; bei Zitaten wird die Absatznummer der Aussage (=A.) im dazugehörigen Interview-Transkript der MAXQDA-Datei angegeben. 
A. 96), zu „kalkulieren“ (I 3, A. 122), zu „wirtschaften“ (I 12, A. 61). Die Domäne beschreibt die Kompetenz, das Geld bzw. die Einnahmen so ,einzuteilen“ (I 14, A. 87; I 24, A. 208; GD 1, A. 127; GD 2, A. 900), dass keine (erneute) Überschuldung entsteht bzw. dass alle Ausgaben bezahlt werden können. Als kompetent in dieser Domäne werden Personen beschrieben, welche die zur Verfügung stehenden finanziellen Mittel mit den Kosten und Ausgaben so abstimmen können, dass „,sie mit dem Geld (...) zurechtkommen“ (I 5, A. 93). Hierbei spielt die Fähigkeit ,planen zu können, also (...) abwägen können“ (I 10, A. 191) eine besondere Rolle. Die Domäne Haushalten beinhaltet die Subdomänen Überblick, Wochen- und Monatsplanung, Jahresplanung, Risiko- und Krisenmanagement sowie Sparen, die unterschiedliche Anforderungen mit sich bringen.

\section{1 .2 Überblick}

Die Kategorie Überblick umfasst die „Fähigkeit, den Überblick über die eigenen Einnahmen und Ausgaben zu behalten, nicht nur zu gewinnen, nicht nur punktuell zu gewinnen, sondern tatsächlich auch im Sinne eines Prozesses den Überblick zu behalten“ (I 1, A. 35). Die Teilnehmenden der Forschenden Lernwerkstatt beschreiben diese Fähigkeit als das „A und O“ beim Umgang mit Geld (GD 2, A. 982). Als Hilfsmittel wird meistens auf das Abheften aller Unterlagen in chronologisch geführten Ordnern oder das Anlegen und Führen eines Haushaltsbuches in Papierform, als PC-Programm oder als App verwiesen (vgl. I 5, A. 47; I 23, A. 50; GB 1; GB 2). Als Negativbeispiel werden in dem Zusammenhang Personen beschrieben, die unsortierte Unterlagen (vgl. I 12, A. 309) oder einen unvollständigen bzw. fehlerhaften Haushaltsplan haben (vgl. I 9, A. 155).

\subsubsection{Wochen- und Monatsplanung}

Wochen und Monatsplanung bezieht sich auf die Gegenüberstellung und Abstimmung der monatlichen Einnahmen und Ausgaben eines Haushalts (vgl. I 13, A. 122; I 17, A. 41; GD 1, A. 1180). Dabei geht es konkret um Prioritätensetzung bei Ausgaben und Kaufentscheidungen, die in Abhängigkeit von den verbleibenden finanziellen Mitteln während des Monats getroffen werden (vgl. I 8, A. 201). Falls finanzielle Mittel in einem Monat nicht ausreichen, müssen beispielsweise bestimmte Käufe auf andere Monate verschoben oder bestimmte Ausgaben reduziert werden (vgl. I 3, A. 63, 555; I 20, A. 111; GD 1, A. 43; GD2, A. 563). Im Hinblick auf solche Ausgaben wie Lebensmittel und Haushaltsbedarf bedeutet die Planung eines Monats eine Verteilung der zur Verfügung stehenden Mittel auf die jeweiligen Wochen (vgl. I 5, A. 93; GB 1, A. 176; GB 2, A. 561). Dies wird in folgender Interviewpassage deutlich:

dass man so ungefähr weiß, was die Sachen im Supermarkt kosten und wie viel ich einkaufen muss, (...) damit das (...) für eine Woche reicht. (...) und am besten eben, dass man schon weiß: Wie viel brauche ich jeden Tag oder wie viel muss ich dann am Ende auch noch haben, damit es so hinkommt? (I 7, A. 219). 


\subsubsection{Jahresplanung}

Die Kategorie Jahresplanung fokussiert die Einplanung der nicht-monatlichen Einnahmen und Ausgaben wie Versicherungen oder Rundfunkgebühren (vgl. I 2, A. 146; I 23, A. 50; GD 1, A. 198; GD 2, A. 36). Vor allem bei einem geringen Einkommen müssen bestimmte Jahres- und Quartalsausgaben auf die Monate verteilt werden, damit die finanzielle Belastung in einem Monat nicht zu hoch ist: ,ganz wesentlicher Aspekt, die (...) jährlichen, halbjährlichen Quartalsposten herunterzubrechen auf den Monat, nicht?"“(I 1, A. 245).

\subsubsection{Risiko- und Krisenmanagement}

Beim Risiko- und Krisenmanagement geht es einerseits um die Berücksichtigung von potenziellen (vorhersehbaren) Kosten wie Stromnachzahlung im Rahmen einer Nebenkostenabrechnung (vgl. I 6, A. 575; GD 1, A. 44). Andererseits beschreibt die Kategorie den kompetenten Umgang mit „unvorhersehbaren Ereignissen“ (I 12, A. 207) bzw. Krisen wie Jobverlust oder Krankheit, die zu Einkommensausfällen bzw. -einbußen führen können (vgl. I 12, A. 147; I 24, A. 182; GD 2, A. 800).

\subsubsection{Sparen}

Die Subdomäne Sparen bezieht sich im Kontext des Haushaltens auf das Zurücklegen einer gewissen Geldsumme, die am Ende des Monats nach Abzug aller Ausgaben eingespart werden kann (vgl. I 4, A. 109; I 24, A. 170; GB 1, A. 13; GD 2, A. 563). Beim geringen Einkommen geht es auch um das Zurücklegen kleiner Beträge, um damit besondere Wünsche und Sonderausgaben zu finanzieren oder sich etwas zusätzlich leisten zu können (vgl. I 6, A. 55; GD 1, A. 28; GD 2, A. 563).

\subsection{Dimensionen}

Die von den drei Expertengruppen genannten Kompetenzanforderungen in den Domänen Finanzieller Grundbildung lassen sich den Kategorien Wissen, Lesen, Schreiben und Rechnen zuordnen.

\subsubsection{Wissen}

Als Voraussetzung für kompetentes Handeln im Bereich Finanzielle Grundbildung wurden in den erhobenen Daten Anforderungen in den Bereichen deklaratives und prozedurales Wissen genannt. So antwortete eine Interviewpartnerin auf die Frage, was zum Umgang mit Geld gehört, folgendermaßen: „Also, erst einmal muss man Wissen haben“ (I 3, A. 136). Beim deklarativen Wissen geht es um Kenntnisse von Sachverhalten wie „Dispo-Zinsen“ (I 2, A. 97), „Einzugsermächtigung“ (I 10, A. 292) oder „Leistungsansprüche“ (I 1, A. 292) sowie Rechte und Pflichten im Bereich Finanzen (vgl. I4, A. 30; GD2, A. 489). Prozedurales Wissen umfasst Anwendungsund Verfahrenswissen - beispielsweise Aspekte wie: 
- Umgang mit Behörden wie Finanzamt, Bundesagentur für Arbeit oder Jobcenter (vgl. I 2, A. 25; GD 2, A. 256 ff.),

- Recherche- und Informationenbeschaffung zu Finanzthemen (vgl. I 8, A. 392; GD 1, A. 615; GD 2, A. 210),

- Nutzung von Online-Vergleichsrechnern (vgl. I 3, A. 265; GD 2; A. 303),

- Vergleich von Angeboten (vgl. I 2, A. 157; I 22, A. 398; GD 2, A. 328).

\subsubsection{Lesen}

Die Dimension Lesen umfasst sinnentnehmendes Lesen von Texten und Dokumenten, die in den verschiedenen Domänen Finanzieller Grundbildung eine Rolle spielen wie Bescheide, Kündigungen, Rechnungen, Verträge und Briefe. Beispielsweise müssen aus Kontoauszügen (vgl. GD 2, A. 344), Stromrechnungen (vgl. I 3, A. 164) und Kreditverträgen (vgl. I 7, A. 300; I 16, A. 71) relevante Informationen entnommen werden. Der Schwierigkeitsgrad beim Lesen hängt mit „,bürokratischen“ (I 3, A. 180) und „komplizierten“ (I 4, A. 56) Formulierungen und Fachbegrifflichkeiten (vgl. I 18, A. 61; GD 1, A. 589; GD 2, A. 32) sowie dem Textaufbau und der Satzlänge zusammen (vgl. I 11, A. 139; I 14, A. 58).

\subsubsection{Schreiben}

In der Kategorie Schreiben werden die Anforderungen zusammengefasst, die das Aufsetzen verschiedenartiger Texte und das Ausfüllen von Anträgen in finanziellen Kontexten umfassen. Als typische Aufgaben im Bereich Schreiben wird das Verfassen von Kündigungen (vgl. GD 2, A. 858), Widerrufsschreiben (vgl. I 5, A. 76; I 9, A. 127) wie auch Briefen oder E-Mails an Behörden (vgl. GD 1, A. 638) genannt. Die verschiedenen Briefempfänger, Textarten und Schreibanlässe erfordern spezifische Formulierungen (vgl. I 18, A. 57; I 20, A. 153) und die Erfüllung bestimmter „Standards“" (I 11, A. 328).

\subsubsection{Rechnen}

Die Kategorie Rechnen umfasst alle Rechenoperationen, die im Handlungsfeld Finanzielle Grundbildung entlang der beschriebenen Domänen relevant sind. So müssen beispielsweise Kosten geschätzt (vgl. I 17, A. 43; I 19, A. 106), finanzielle Mittel nach Abzug aller Ausgaben berechnet (vgl. GD 2, A. 561) oder Preise verglichen (vgl. I 4, A. 87) werden. In der Regel geht es dabei nicht um die mathematische Genauigkeit, sondern um eine grobe Abschätzung bzw. eine Überschlagsrechnung (vgl. I 6, A. 597; I 12, A. 279; GD 2, A. 889).

\section{3 (Handlungs-)Anforderungen in Alltagssituationen}

Als Grundlage für die Entwicklung von Programmen und Angeboten in der Erwachsenenbildung beinhaltet das Modell nicht nur die sechs Kompetenzdomänen und vier Dimensionen als Grundkategorien, sondern auch exemplarische (Handlungs-)Anfor- 


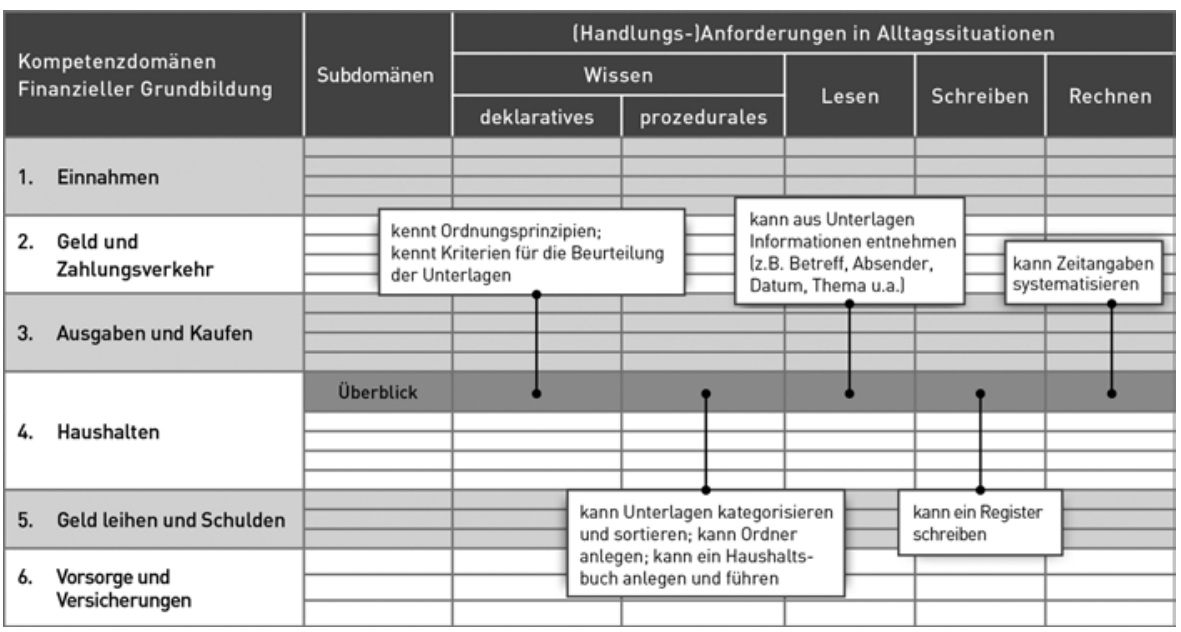

Abb. 1 Grundzüge des Kompetenzmodells Finanzielle Grundbildung

derungen, aus denen lebensweltorientierte Lernziele und -inhalte abgeleitet werden können.

Bei der Formulierung der konkreten Anforderungen konnte zum einen auf die Beispiele in dem empirischen Material zurückgegriffen werden. Da die Datenerhebung nicht auf die Vollständigkeit der Erfassung der konkreten Kompetenzanforderungen zielte, sondern auf die Erhebung der Grundelemente, wurden zum anderen weitere beispielhafte (Handlungs-)Anforderungen im Sinne der oben beschriebenen Heuristik formuliert. Dabei wurde im Sinne einer ,konsensuellen“ (Grotlüschen et al. 2014, S. 51) Entwicklung von Kompetenzmodellen und einer kommunikativen Validierung auf die Expertise der Kooperationspartnerinnen und -partner des Projekts aus den Feldern Schuldnerberatung und Erwachsenenbildung zurückgegriffen, unter anderem in Form einer Fokusgruppe in der Schuldnerberatung des Sozialdienstes Katholischer Männer in Köln.

Das vollständige Kompetenzmodell beinhaltet entlang aller Domänen und Dimensionen Finanzieller Grundbildung beispielhafte (Handlungs-)Anforderungen in Alltagssituationen bzw. Kann-Beschreibungen, ${ }^{3}$ die im Folgenden exemplarisch für die Domäne Haushalten, Subdomäne Überblick, genannt werden (vgl. Abb. 1):

- Deklaratives Wissen: kennt Ordnungsprinzipien; kennt Kriterien für die Beurteilung der Unterlagen;

- Prozedurales Wissen: kann Unterlagen kategorisieren und sortieren; kann Ordner anlegen; kann ein Haushaltsbuch anlegen und führen;

- Lesen: kann aus Unterlagen Informationen entnehmen (z. B. Betreff, Absender, Datum, Thema u. a.);

- Schreiben: kann ein Register schreiben;

- Rechnen: kann Zeitangaben systematisieren.

\footnotetext{
${ }^{3}$ Das vollständige Modell kann auf der Projekt-Homepage (www.die-curve.de) heruntergeladen werden.
} 
Kompetentes Handeln in einem bestimmten inhaltlichen Feld Finanzieller Grundbildung erfordert in der Regel Kenntnisse und Fähigkeiten in allen vier Dimensionen, wobei gerade das deklarative Wissen prinzipiell die Voraussetzung für die Erfüllung der Anforderungen in den restlichen Dimensionen darstellt. Das Modell ist nicht hierarchisch aufgebaut, sondern enthält Aufgaben mit unterschiedlichem Schwierigkeitsgrad innerhalb des niedrigsten Kompetenzlevels, des sogenannten Level-One.

\section{Fazit und Ausblick}

Das entwickelte Kompetenzmodell stellt erstmalig eine theoretisch und empirisch fundierte Grundlage für die Strukturierung und Bestimmung von Inhalten bei der Entwicklung von Angeboten im Bereich Finanzielle Grundbildung dar. In Anlehnung an die Konzepte zu Literalität als soziale Praxis werden Lesen, Schreiben und Rechnen nicht als kontextlose Grundbildungskompetenzen vorausgesetzt, sondern sind integrale Bestandteile des Modells. Unter Berücksichtigung des jeweiligen Einrichtungsprofils, der Adressatinnen und Adressaten sowie der Kooperationspartnerinnen und -partner können verschiedene Angebotskonzepte entstehen, die entweder alle Elemente des Modells beinhalten oder ausgewählte Kategorien fokussieren (vgl. Tröster und Mania 2015).

Kompetenzmodelle, die von Verwendungssituationen im Alltag ausgehen und unter Beteiligung potenzieller Lernender entstanden sind, entsprechen den Prinzipen der Lebenswelt- und Teilnehmerorientierung, denen im Rahmen einer Didaktik der Grundbildung eine besondere Rolle zugeschrieben wird (vgl. Brödel 2012). Das Modell dient als didaktische Grundlage für Programmplanende und Lehrende in der Erwachsenenbildung. Dabei geht es nicht um die Hierarchisierung und Curricularisierung von Inhalten im Sinne von starren Vorgaben an die Bildungseinrichtungen, welche die Angebotsvielfalt einschränken würden, sondern um die Unterstützung des pädagogischen Handelns als Beitrag zur Professionalisierung und Ausdifferenzierung verschiedener Grundbildungsbereiche.

Mit Blick auf Forschungsdesiderata wäre vor dem Hintergrund der Entwicklung des Kompetenzmodells vor allem im Kontext der Schuldnerberatung und Überschuldung eine Überprüfung bzw. Validierung des Modells mit weiteren Adressatengruppen der (Finanziellen) Grundbildung sinnvoll. Für die Konzeption von (aufeinander aufbauenden) Lernangeboten könnten auch die Schwierigkeitsgrade der beschriebenen Anforderungen bestimmt werden. Im Rahmen der Diskussion um biografisches Lernen und Übergänge als Lernanlässe (vgl. Hof et al. 2014) wäre die Bedeutung der verschiedenen Themen Finanzieller Grundbildung im Verlauf des Lebens sowie der Einfluss der nicht-kognitiven Kompetenzbestandteile auf das kompetente Handeln im Bereich Umgang mit Geld zu untersuchen. Nicht ausreichend wurde bisher auch die Frage nach der Überschneidung mit anderen Grundbildungsbereichen wie Food Literacy, Computer Literacy oder der politischen Grundbildung beantwortet.

Open Access Dieser Artikel wird unter der Creative Commons Namensnennung 4.0 International Lizenz (http://creativecommons.org/licenses/by/4.0/deed.de) veröffentlicht, welche die uneingeschränkte Nutzung, Verbreitung und Wiedergabe für beliebige Zwecke erlaubt, sofern Sie den/die ursprünglichen Autor(en) und die Quelle ordnungsgemäß nennen, einen Link zur Creative Commons Lizenz beifügen und angeben, ob Änderungen vorgenommen wurden. 


\section{Literatur}

Ambos, I., \& Greubel, S. (2012). Ökonomische Grundbildung. Themenfeld „Akteurs- und Angebotsanalyse“. Abschlussbericht. http://www.die-bonn.de/doks/2012-oekonomische-grundbildung-akteursund-angebotsanalyse-01.pdf. Zugegriffen: 30. März 2015.

Aprea, C. (2012). Messung der Befähigung zum Umgang mit Geld und Finanzthemen. Ausgewählte Instrumente und alternative diagnostische Zugänge. Berufs- und Wirtschaftspädagogik - online, 22. http://www.bwpat.de/ausgabe22/aprea_bwpat22.pdf. Zugegriffen: 30. März 2015.

Arnold, R. (2010). Didaktik - Methodik. In R. Arnold, S. Nolda, \& E. Nuissl (Hrsg.), Wörterbuch Erwachsenenbildung (S. 64-66). Bad Heilbrunn: Klinkhardt.

Barton, D., \& Hamilton, M. (2003). Situated literacies. Reading and writing in context. London: Routledge. (EA 2000).

Baumert, J., \& Kunter, M. (2006). Stichwort: Professionelle Kompetenz von Lehrkräften. Zeitschrift für Erziehungswissenschaft, 9(4), 469-520.

BMBF - Bundesministerium für Bildung und Forschung. (2012). Vereinbarung über eine gemeinsame nationale Strategie für Alphabetisierung und Grundbildung Erwachsener in Deutschland 20122016. Berlin. http://www.bmbf.de/pubRD/NEU_strategiepapier_nationale_alphabetisierung.pdf. Zugegriffen: 30. März 2015.

Bremer, H., \& Kleemann-Göhring, M. (2011). Weiterbildung und „Bildungsferne“. Forschungsbefunde, theoretische Einsichten und Möglichkeiten für die Praxis. Essen. http://www.uni-due.de/imperia/md/ content/politische-bildung/arbeitshilfe_potenziale. Zugegriffen: 30. März 2015.

Brödel, R. (2012). Didaktik der Grundbildung. Plädoyer für einen lebensweltorientierten Ansatz. Erwachsenenbildung, 2, 63-66.

Egloff, B. (2014). Grundbildung - Zur Einführung in den Themenschwerpunkt. Hessische Blätter für Volksbildung, 2, 103-106.

Elliott, A., Dolan, P., Vlaev, I., Adriaenssens, C., \& Metcalfe, R. (2010). Transforming Financial Behaviour: developing interventions that build financial capability. A Report for the Consumer Financial Education Body. http://pauldolan.co.uk/wp-content/uploads/2011/05/20100713_transforming_financial_behaviour.pdf. Zugegriffen: 30. März 2015.

Flick, U. (1995). Stationen des qualitativen Forschungsprozesses. In U. Flick, E. von Kardoff, H. Keupp, L. von Rosenstiel, \& S. Wolff (Hrsg.), Handbuch qualitative Forschung: Grundlagen, Konzepte, Methoden und Anwendungen (S. 148-171). Weinheim: Beltz.

Frank, S. (2013). Kompetente Bildung oder eingebildete Kompetenz? Kompetenzen als inhaltsdidaktische Leitgröße. Magazin erwachsenenbildung.at, 20, 04-1-04-7. http://www.erwachsenenbildung.at/ magazin/13-20/meb13-20.pdf. Zugegriffen: 30. März 2015.

Freire, P. (1974). Erziehung als Praxis der Freiheit. Stuttgart: Kreuz-Verlag.

Gieseke, W. (2008). Bedarfsorientierte Angebotsplanung in der Erwachsenenbildung. Bielefeld: W. Bertelsmann.

Gläser, J., \& Laudel, G. (2004). Experteninterviews und qualitative Inhaltsanalyse als Instrumente rekonstruktiver Untersuchungen. Wiesbaden: VS Verlag für Sozialwissenschaften.

Gomez Tutor, C. (2010). Generative Themen. In R. Arnold, S. Nolda, \& E. Nuissl (Hrsg.), Wörterbuch Erwachsenenbildung (S. 124-125). Bad Heilbrunn: Klinkhardt.

Grell, P. (2006). Forschende Lernwerkstatt. Eine qualitative Untersuchung zu Lernwiderständen in der Weiterbildung. Münster: Waxmann.

Grotlüschen, A., \& Riekmann, W. (Hrsg.). (2012). Funktionaler Analphabetismus in Deutschland. Ergebnisse der ersten leo. - Level-One Studie. Münster: Waxmann.

Grotlüschen, A., Bonna, F., Euringer, C., \& Heinemann, A. M. B. (2014). Konsequenzen der Konstruktion von Literalität hinsichtlich der Vergleichbarkeit der Alpha-Levels mit den Niveaustufen des Europäischen Referenzrahmens Sprachen. In H. Pätzold, H. von Felden, \& S. Schmidt-Lauff (Hrsg.), Programme, Themen und Inhalte in der Erwachsenenbildung (S. 51-65). Baltmannsweiler: Schneider.

von Hippel, A., Fuchs, S., \& Tippelt, R. (2008). Weiterbildungsorganisationen und Nachfrageorientierung - neo-institutionalistische Perspektiven. Zeitschrift für Pädagogik, 54(5), 663-678.

Hof, C., Meuth, M., \& Walther, A. (2014). Pädagogik der Übergänge. Übergänge in Lebenslauf und Biografie als Anlässe und Bezugspunkte von Erziehung, Bildung und Hilfe. Weinheim: Beltz Juventa.

Höffer-Mehlmer, M. (2011). Programmplanung und -organisation. In R. Tippelt \& A. von Hippel (Hrsg.), Handbuch Erwachsenenbildung/Weiterbildung (S. 989-1002). Wiesbaden: VS Verlag für Sozialwissenschaften. 
Hummelsheim, S. (2010). Ökonomische Grundbildung tut not: Empirische Studien weisen auf erhebliche Defizite in der ökonomischen und finanziellen Grundbildung hin. DIE Fakten. http://www.die-bonn. de/doks/hummelsheim1001.pdf. Zugegriffen: 30. März 2015.

Klieme, E., \& Leutner, D. (2006). Kompetenzmodelle zur Erfassung individueller Lernergebnisse und zur Bilanzierung von Bildungsprozessen. Überarbeitete Fassung des Antrags an die DFG auf Einrichtung eines Schwerpunktprogramms. http://kompetenzmodelle.dipf.de/pdf/rahmenantrag. Zugegriffen: 30. März 2015.

Lehner, M. (2013). Inhalte als zentrale Aspekte einer Didaktik der Erwachsenenbildung. Magazin erwachsenenbildung.at, 20, 03-1-03 - 10. http://www.erwachsenenbildung.at/magazin/13-20/meb13-20. pdf. Zugegriffen: 30. März 2015.

Leinert, J. (2004). Nachhilfe zur Vorsorge. Schlechte finanzielle Allgemeinbildung. Die Zeitschrift für Erwachsenenbildung, 4, 45-47.

Mania, E., \& Tröster, M. (2014). Finanzielle Grundbildung - Ein Kompetenzmodell entsteht. Hessische Blätter für Volksbildung, 2, 136-145.

OECD - Organisation for Economic Co-operation and Development. (2014). PISA 2012 Results: Students and Money: Financial Literacy Skills for the 21st Century (Volume VI), PISA. OECD Publishing. http://www.oecd.org/pisa/keyfindings/PISA-2012-results-volume-vi.pdf. Zugegriffen: 30. März 2015.

Piorkowsky, M. B. (2009). Lernen, mit Geld umzugehen. Aus Politik und Zeitgeschichte, 26, 40-46, Bonn. http://www.bpb.de/system/files/pdf/QNPDYV.pdf. Zugegriffen: 30. März 2015.

Rammstedt, B. (Hrsg.). (2013). Grundlegende Kompetenzen Erwachsener im internationalen Vergleich: Ergebnisse von PIAAC 2012. Münster: Waxmann. http://nbn-resolving.de/urn:nbn:de:0168ssoar-360687. Zugegriffen: 30. März. 2015.

Reich-Claassen, J., \& von Hippel, A. (2011). Angebotsplanung und -gestaltung. In R. Tippelt \& A. von Hippel (Hrsg.), Handbuch Erwachsenenbildung/Weiterbildung (S. 1003-1015). Wiesbaden: VS Verlag für Sozialwissenschaften.

Reifner, U. (2006). Financial literacy in Europe. Baden-Baden: Nomos.

Remmele, B., Seeber, G., Speer, S., \& Stoller, F. (2013). Ökonomische Grundbildung für Erwachsene. Ansprüche - Kompetenzen - Grenzen. Schwalbach/Ts.: Wochenschau.

Schemmann, M., \& Seitter, W. (2015). Angebotsentwicklung in der wissenschaftlichen Weiterbildung als Resultate eines vierfachen Zielgruppenbezugs. In H. Pätzold, H. von Felden, \& S. Schmidt-Lauff (Hrsg.), Programme, Themen und Inhalte in der Erwachsenenbildung (S. 154-169). Baltmannsweiler: Schneider.

Schlutz, E. (2005). Didaktischer Epochenwechsel? Klärungsbedarfe zur Weiterentwicklung des didaktischen Denkens, mit Blick auf ein DFG-gefördertes Forschungsvorhaben. Report: Zeitschrift für Weiterbildungsforschung, 28(3), 18-26.

Schlutz, E. (2006). Bildungsdienstleistungen und Angebotsentwicklung. Münster: Waxmann.

Schrader, J. (2010). Mediengestützte Fallarbeit. Grundlagen und Zielsetzungen eines Forschungs- und Entwicklungsprojekts zur Kompetenzentwicklung von Lehrenden in der Erwachsenenbildung. In J. Schrader, R. Hohmann, \& S. Hartz (Hrsg.), Mediengestützte Fallarbeit. Konzepte, Erfahrungen und Befunde zur Kompetenzentwicklung von Erwachsenenbildnern (S. 71-124). Bielefeld: W. Bertelsmann.

Schürkmann, S., \& Schuhen, M. (2013). Kompetenzmessung im Bereich financial literacy. Ergebnisse zum Umgang mit Online-Rechnern aus der FILS-Studie. Zeitschrift für ökonomische Bildung, 01, 73-89. http://zfoeb.de/2013_1/schuerkmann_schuhen.pdf. Zugegriffen: 30. März. 2015.

Seeber, G., Retzmann, T., Remmele, B., \& Jongebloed, H.-C. (2012). Bildungsstandards der ökonomischen Allgemeinbildung. Kompetenzmodell-Aufgaben - Handlungsempfehlungen. Schwalbach/Ts.: Wochenschau.

Siebert, H. (1982). Programmplanung als didaktisches Handeln. In E. Nuissl (Hrsg.), Taschenbuch der Erwachsenenbildung. Baltmannsweiler: Schneider.

Siebert, H. (2005). Didaktik - mehr als die Kunst des Lehrens? Report. Zeitschrift für Weiterbildungsforschung, (28)3, 9-17.

Siebert, H. (2006). Didaktisches Handeln in der Erwachsenenbildung. Didaktik aus konstruktivistischer Sicht (5., überarb. Aufl). Augsburg: Ziel.

Siebert, H. (2010). Curriculum. In R. Arnold, S. Nolda, \& E. Nuissl (Hrsg.), Wörterbuch Erwachsenenbildung (S. 59). Bad Heilbrunn: Klinkhardt.

Street, B. V. (2003). What's „new“ in new literacy studies? Current Issues in Comparative Education, 5(2), 77-91. 
The Basic Skills Agency and Financial Services Authority. (2006). Financial Capability in the UK: Delivering Change. http:/www.fsa.gov.uk/static/pubs/other/fincap_delivering.pdf. Zugegriffen: 30. März 2015.

The New London Group. (1996). A pedagogy of multiliteracies: Designing social futures. Harvard Educational Review, 66(1), 60-92.

Tröster, M., \& Mania, E. (2015). Entwicklung neuer Angebotsformate im Bereich Finanzielle Grundbildung als Dialog zwischen Sozialer Arbeit und Erwachsenenbildung. ALFA-Forum, 87, 32-35.

Weber, B., Eik, I. van, \& Maier, P. (Hrsg.). (2013). Ökonomische Grundbildung für Erwachsene. Ansprüche und Grenzen, Zielgruppen, Akteure und Angebote - Ergebnisse einer Forschungswerkstatt. Bielefeld: W. Bertelsmann.

Weinert, F. E. (2001). Vergleichende Leistungsmessung in Schulen. In F. E. Weinert (Hrsg.), Leistungsmessung in Schulen (S. 17-31). Weinheim: Beltz.

Zeuner, C., \& Pabst, A. (2011). „Lesen und Schreiben eröffnen eine neue Welt! “ Literalität als soziale Praxis - Eine ethnographische Studie. Bielefeld: W. Bertelsmann. 